\title{
ON CONVERSE GAP THEOREMS
}

BY

\section{GEORGE POLYYA}

1. In what follows, I consider power series with preassigned vanishing coefficients. I write such a series in the form

$$
a_{1} z^{\lambda_{1}}+a_{2} z^{\lambda_{2}}+\cdots+a_{n} z^{\lambda_{n}}+\cdots \cdot
$$

The numbers $a_{n}$ are different from 0 and the $\lambda_{n}$ are integers,

$$
0<\lambda_{1}<\lambda_{2}<\cdots<\lambda_{n}<\lambda_{n+1}<\cdots \text {. }
$$

I assume that the radius of convergence of the series (1) is finite and different from 0 .

I quote two well known theorems ${ }^{(1)}$.

TheOREM I. If

$$
\lim _{n \rightarrow \infty} n \lambda_{n}^{-1}=0
$$

the domain of existence of the analytic function defined by (1) is the interior of the circle of convergence of the series (1).

THEOREM II. If

$$
\underset{n \rightarrow \infty}{\liminf } n \lambda_{n}^{-1}=0
$$

the domain of existence of the analytic function defined by (1) is a simply connected part of the $z$-plane (from which it follows that the function defined by (1) is uniform).

Is it possible to improve these theorems by enlarging the hypothesis? Is there a less exacting hypothesis leading to the same conclusion? I say no. In fact I shall solve the following problems $\left({ }^{2}\right)$.

Presented to the Society, February 22, 1941 ; received by the editors June 4, 1941.

(1) In the following the two parts of my paper Untersuchungen über Lïcken und Singularitäten von Potenzreihen, Mathematische Zeitschrift, vol. 29 (1929), pp. 549-640 and Annals of Mathematics, (2), vol. 34 (1933), pp. 731-777 will be quoted as LS I and LS II. For Theorem I (Fabry's theorem) see LS I, p. 627, Theorem VIa; for Theorem II (theorem of the present author) see LS II, p. 737, Theorem B.

(2) These problems have been stated, with an indication of the proof: I in Comptes Rendus de l'Académie des Sciences, Paris, vol. 208 (1939), pp. 709-711; II in the Bulletin of the American Mathematical Society, vol. 47 (1941), p. 207. A problem related to I was stated by G. Szegö, Acta Litterarum ac Scientiarum Szeged, vol. 1 (1923), p. 73. 
Problem I. Given an infinite sequence of integers $\lambda_{1}, \lambda_{2}, \cdots, \lambda_{n}, \cdots$ satisfying (2) but not satisfying (3), find a power series of the form (1) defining an analytic function whose domain of existence extends beyond the circle of convergence.

PROBLEM II. Given an infinite sequence of integers $\lambda_{1}, \lambda_{2}, \cdots, \lambda_{n}, \cdots$ satisfying (2) but not satisfying (4), find a power series of the form (1) defining a multiform analytic function (whose domain of existence, consequently, cannot be a simply connected part of the z-plane).

2. It is of some interest to restate the facts which we proposed to prove in a different terminology. This terminology is due to Borel but, so far as I know, it was used by him just once $\left({ }^{3}\right)$ and it has never been used since.

Let us consider power series whose radius of convergence is different from 0 and $\infty$ and let us say that two such series

$$
\sum_{0}^{\infty} c_{n} z^{n}, \quad \sum_{0}^{\infty} c_{n}^{\prime} z^{n}
$$

belong to the same class, if they have the same distribution of nonvanishing coefficients, that is if, for $n=0,1,2, \cdots, c_{n}$ and $c_{n}^{\prime}$ are either both 0 or both different from 0 . In fact, a class of power series is characterized by those powers of $z$ whose coefficients do not vanish, and therefore by an increasing sequence of integers $\lambda_{1}, \lambda_{2}, \cdots$ and all series belonging to the class have the same form $(1)\left({ }^{4}\right)$. Let us call

$$
\lim _{n \rightarrow \infty} n \lambda_{n}^{-1}, \quad \liminf _{n \rightarrow \infty} n \lambda_{n}^{-1}, \quad \limsup _{n \rightarrow \infty} n \lambda_{n}^{-1}
$$

(in the given order) the density, the lower density and the upper density of the class (the first may not exist, but the second and the third necessarily exist). With this terminology, we may compound Theorem I and Problem I into one short statement (and the same for Theorem II and Problem II):

I. In order that all power series of a class have their circle of convergence as natural boundary it is necessary and sufficient that the density of the class be 0 .

II. In order that all power series of a class define uniform analytic functions it is necessary and sufficient that the lower density of the class be 0.

A few other well known facts on the singularities of power series may be quite elegantly stated in the same terminology $\left({ }^{5}\right)$.

(3) Comptes Rendus de l'Académie des Sciences, Paris, vol. 137 (1903), pp. 695-697.

(4) Series (1) corresponds to the (evidently unessential) assumption that the coefficient of $z^{0}$ is zero in all series of the class.

(5) See also, for further literature, LS I, p. 622, Theorem IIIa for the first, and LS II, p. 745, Theorem IV for the third statement. The second statement is easy. 
Each class contains non-continuable power series.

In order that a class contain a power series having on its circle of convergence a pole and no other singular point, it is necessary and sufficient, that the sequence $\lambda_{1}, \lambda_{2}, \cdots$ contain nearly all integers, that is, either all integers or all with a finite number of exceptions.

In order that a class contain a power series having on its circle of convergence an essential singular point (an isolated singularity which is not a pole and not a branch point) and no other singular point, it is necessary and sufficient that the density of the class exist and be 1.

These theorems seem to suggest that there are a few more of the same kind.

3. Our solution of the Problems I and II uses some properties of the series

$$
F(1) z+F(2) z^{2}+\cdots+F(m) z^{m}+\cdots=\Phi(z)
$$

where $F(z)$ denotes an entire function of exponential type $\left(^{6}\right)$. The real-valued periodic function $h(\phi)$ of the real variable $\phi$, defined by

$$
h(\phi)=\limsup _{r \rightarrow \infty} r^{-1} \log \left|F\left(r e^{i \phi}\right)\right|
$$

is called the indicator of $F(z)$. The series (5) has a finite radius of convergence and its analytic continuation is closely connected with the indicator $h(\phi)$. I quote the following fact $\left({ }^{7}\right)$ :

If

$$
h(-\pi / 2)<\pi, \quad h(\pi / 2)<\pi
$$

then the function $\Phi(z)$ defined by the series (5) is regular along the negative real axis, and also at the point $z=\infty$ in whose neighborhood it has the development

$$
\Phi(z)=-F(0)-\frac{F(-1)}{z}-\frac{F(-2)}{z^{2}}-\cdots .
$$

This theorem yields a quick solution of Problem II. In fact, assume that the sequence of integers $\lambda_{1}, \lambda_{2}, \cdots$ does not satisfy (4). Then there exists a positive $\alpha$, such that

$$
\frac{n}{\lambda_{n}}>\alpha
$$

Define

$$
G(z)=\prod_{n=1}^{\infty}\left(1-\frac{z^{2}}{\lambda_{n}^{2}}\right) .
$$

( ${ }^{(6)}$ Defined in LS I, p. 578.

(7) See LS I, pp. 604-609, especially formulae (72) and (73), p. 609. Observe that the $\Phi(z)$ of these formulae differs by an additive constant from the $\Phi(z)$ of the present formula (5). 
It follows from (9), (10) that, for $r>0$,

$$
G(i r)=\prod_{n=1}^{\infty}\left(1+\frac{r^{2}}{\lambda_{n}^{2}}\right)>\prod_{n=1}^{\infty}\left(1+\frac{\alpha^{2} r^{2}}{n^{2}}\right)=\frac{\sin i \pi \alpha r}{i \pi \alpha r}=\frac{e^{\pi \alpha r}-e^{-\pi \alpha r}}{2 \pi \alpha r} .
$$

Define $F(z)$ by the equation

$$
\pi z F(z) G(z)=\sin \pi z .
$$

$F(z)$ is evidently an entire function of exponential type $\left({ }^{8}\right)$. For positive integral $m$

$$
\begin{aligned}
& F(m)=\left\{\begin{array}{ccc}
(-1)^{\lambda_{n}} /\left(\lambda_{n} G^{\prime}\left(\lambda_{n}\right)\right) & \text { if } & m=\lambda_{n} \\
0 & \text { if } & m \neq \lambda_{n}
\end{array}\right. \\
& F(0)=1,
\end{aligned}
$$

and, by virtue of (11) and (12),

$$
\limsup _{r \rightarrow \infty} r^{-1} \log |F( \pm i r)| \leqq \pi-\pi \alpha .
$$

Consider the power series, arising from (5),

$$
\int_{0}^{z} \Phi(z) z^{-1} d z=\sum_{1}^{\infty} \frac{F(m) z^{m}}{m}=\sum_{n=1}^{\infty} \frac{(-1)^{\lambda_{n}} z^{\lambda_{n}}}{\lambda_{n}^{2} G^{\prime}\left(\lambda_{n}\right)} .
$$

Series (16) is evidently of form (1). The condition (7) is fulfilled, see (6) and (15). Therefore, the analytic continuation of (16) along the negative real axis is possible. But, in a certain neighborhood of the point $z=\infty$, we have, by virtue of (8) and (14), with a certain constant $C$,

$$
\int_{0}^{z} \Phi(z) z^{-1} d z=C-\log z+\frac{F(-1)}{z}+\frac{F(-2)}{2 z^{2}}+\cdots
$$

and therefore the analytic continuation of (16) is not a single-valued function. Thus (16) fulfills all the requirements of Problem II.

4. Now we are going to solve Problem I. We use again the series (5) and the connection between the analytic continuation of this series and the indicator $h(\phi)$. We need now the following facts $\left({ }^{9}\right)$.

To each entire function $F(z)$ of exponential type corresponds a bounded and closed convex domain $\mathrm{J}$, called the indicator diagram of $F(z)$. The domain $\mathrm{J}$ lies in the half-plane

$$
x \cos \phi+y \sin \phi-h(\phi) \leqq 0
$$

(8) In fact, (24) holds also for the present $F(z)$.

(9) See LS I, pp. 604-609, especially p. 606. (Correct the misprint at the end of the fqurteenth line from the top of p. 606; read $h(0)$ instead of 0 .) I write as usual $z=x+i y$ with real $x, y$ in the following statement and later in (26), and (27). 
but has a point on the boundary of this half-plane; this holds for all real $\phi$. If the circle of convergence of the power series (5) is a natural boundary, the boundary of the indicator diagram $\mathfrak{J}$ of $F(z)$ contains a segment of a vertical straight line, of length not less than $2 \pi$, limiting 3 from the right.

We shall use this fact at the end of the following construction which we divide in successive steps.

i. We start from a sequence $\lambda_{1}, \lambda_{2}, \cdots$ that does not satisfy (3). It follows that, for a fixed $\alpha, 0<\alpha<1$, there are arbitrarily distant intervals of the form $r \alpha<x<r$ which contain more than $r \delta$ points of the sequence $\lambda_{1}, \lambda_{2}, \cdots, \delta$ being positive, sufficiently small, but fixed $\left({ }^{10}\right)$. Thus we can choose a sequence of increasing positive integers $n_{1}, n_{2}, \cdots$ and a positive $\delta$, satisfying the following condition :

(I) The interval between $\left(n_{k}-1 / 2\right) / 2^{1 / 2}$ and $n_{k}-1 / 2$, which I denote by $I_{k}$, contains at least $\delta n_{k}$ points of the sequence $\lambda_{1}, \lambda_{2}, \cdots$.

By rejecting if necessary certain elements of the chosen sequence, we can find a sequence (a subsequence of the first chosen sequence, which, by an appropriate change of notation, will be called again $n_{1}, n_{2}, \cdots$ ) satisfying not only condition (I) but also the following:

$$
\begin{gathered}
n_{k-1}<\left(n_{k}-\frac{1}{2}\right) / 2^{1 / 2} \\
\log \left[\left(\frac{n_{k}}{1}\right)^{2}-1\right]\left[\left(\frac{n_{k}}{2}\right)^{2}-1\right] \cdots\left[\left(\frac{n_{k}}{n_{k-1}}\right)^{2}-1\right]<\left(n_{k}-\frac{1}{2}\right)^{1 / 2} .
\end{gathered}
$$

In fact, $n_{k-1}$ being fixed, both inequalities (II) are satisfied by any sufficiently great $n_{k}$.

Call those elements of the sequence $\lambda_{1}, \lambda_{2}, \ldots$ which are contained in the intervals $I_{1}, I_{2}, \cdots, I_{k}, \cdots$, numbered in order of magnitude, $\mu_{1}, \mu_{2}, \mu_{3}, \cdots$.

ii. Using the sequence $\mu_{1}, \mu_{2}, \cdots$ we just constructed, define

$$
G(z)=\prod_{n=1}^{\infty}\left(1-\frac{z^{2}}{\mu_{n}^{2}}\right) .
$$

This definition is different from (10), which we used in solving Problem II, and which we disregard now. We shall estimate

$$
\begin{aligned}
\left(n_{k}-\frac{1}{2}\right)^{-1} \log \left|G\left(n_{k}-\frac{1}{2}\right)\right| & =\sum_{j=1}^{\infty}\left(n_{k}-\frac{1}{2}\right)^{-1} \log \left|\left(\frac{n_{k}-\frac{1}{2}}{\mu_{j}}\right)^{2}-1\right| \\
& =S_{1}+S_{2}+S_{3}
\end{aligned}
$$

$S_{2}$ contains those terms of the sum on the right-hand side of the first line whose $\mu_{j}$ is contained in $I_{k} ; S_{1}$ those terms whose $\mu_{j}$ is contained in one of

(10) The easy proof can be left to the reader; it is contained in the fuller developments of LS I, pp. 556-560, especially in (14) p. 559. 
the intervals $I_{1}, I_{2}, \cdots, I_{k-1} ; S_{3}$ those whose $\mu_{j}$ is in one of the intervals $I_{k+1}, I_{k+2}, \cdots$. These intervals do not overlap, by the first inequality (II). If $\mu_{j}$ is in $S_{1}$ then $\mu_{j}<n_{k-1}-1 / 2$ and therefore, by the second inequality (II),

$$
S_{1}<\left(n_{k}-\frac{1}{2}\right)^{-1 / 2} \rightarrow 0
$$

as $n_{k} \rightarrow \infty$. If $\mu_{j}$ is in $S_{3}$ then $\mu_{j}>n_{k}-1 / 2$ and therefore each term in $S_{3}$ is negative,

$$
S_{3}<0 \text {. }
$$

The terms in $S_{2}$ are also negative, and in number not less than $n_{k} \delta$, by condition (I); the single term decreases algebraically (increases in absolute value) as $\mu_{j}$ increases. We obtain the (algebraically) greatest value of $S_{\mathbf{2}}$ by taking as few terms as possible and terms as close to the left-hand end point of $I_{k}$ as possible. If the summation is extended to the integers $l$ satisfying.

$$
\left(n_{k}-\frac{1}{2}\right) / 2^{1 / 2}<l<\left(n_{k}-\frac{1}{2}\right) / 2^{1 / 2}+n_{k} \delta
$$

we have

$$
\begin{aligned}
S_{2} & <\sum\left(n_{k}-\frac{1}{2}\right)^{-1} \log \left[\left(\frac{n_{k}-\frac{1}{2}}{l}\right)^{2}-1\right] \\
& \rightarrow \int_{1 / 2^{1 / 2}}^{(1 / 21 / 2)+\delta} \log \left(\frac{1}{t^{2}}-1\right) d t<0 .
\end{aligned}
$$

By (18), (19), (20), (21) we obtain, $n$ running through the positive integers, that

$$
\liminf _{n \rightarrow \infty}\left(n-\frac{1}{2}\right)^{-1} \log \left|G\left(n-\frac{1}{2}\right)\right|<0 .
$$

iii. Define $F(z)$ by (12). From (12) and (22) it follows that

$$
\begin{aligned}
h(0) & =\limsup _{r \rightarrow \infty} r^{-1} \log |F(r)| \\
& \geqq \limsup _{n \rightarrow \infty}\left(n-\frac{1}{2}\right) \log \frac{1}{\pi\left(n-\frac{1}{2}\right)\left|G\left(n-\frac{1}{2}\right)\right|}>0 .
\end{aligned}
$$

On the other hand, if $|z|=r$,

$$
|F(z)| \leqq F(i r)<\prod_{n=1}^{\infty}\left(1+\frac{r^{2}}{n^{2}}\right)=\frac{e^{\pi r}-e^{-\pi r}}{2 \pi r}
$$

and therefore, by (6), for all real $\phi$

$$
h(\phi) \leqq \pi .
$$

Inequalities for $h(\phi)$ are equivalent to geometric conditions for $J$, the indicator diagram of $F(z)$. In the present case, $F(z)$ is an even function and real- 
valued for real $z$; so $J$ is symmetrical with respect to the real and to the imaginary axes. By (25), $J$ is contained in the circle

$$
x^{2}+y^{2}=\pi^{2} .
$$

By (23), it has a common point with the vertical line

$$
x=h(0)
$$

and no point with an abscissa greater then $h(0)$. But the segment, intercepted on the line (27) by the circle (26) is shorter than $2 \pi$, because, by $(23), h(0)>0$; there is no segment of a vertical line, of length greater than or equal to $2 \pi$, on the boundary of the indicator diagram $\mathfrak{J}$.

iv. This last result shows that the series (5)

$$
\Phi(z)=\sum_{1}^{\infty} F(m) z^{m}=\sum_{n=1}^{\infty} \frac{(-1)^{\mu_{n}} z^{\mu_{n}}}{\mu_{n} G^{\prime}\left(\mu_{n}\right)}
$$

is continuable, by virtue of the fact quoted at the beginning of this section. Series (28) is not exactly of the form (1), because some of the coefficients $a_{n}$ may be zero, if $\mu_{1}, \mu_{2}, \cdots$ is a proper subsequence of $\lambda_{1}, \lambda_{2}, \cdots$ and not identical with the latter. If the coefficient of $z^{\lambda_{n}}$ in (28) is 0 , add the term

$$
\frac{z^{\lambda_{n}}}{\lambda_{n} !}
$$

to (28). The series obtained in this way is exactly of the form (1) and satisfies all requirements of Problem I.

Brown UNIVERSITY,

Providence, R. I. 